$>$ Plusieurs traitements modifiant l'évolution de la sclérose en plaques (SEP) ont fait leur apparition ces dernières années. Par rapport aux traitements plus anciens, les nouvelles molécules, déjà approuvées ou en cours d'essai clinique, ont un profil d'efficacité habituellement supérieur et/ ou un mode d'administration plus simple, notamment pour les traitements oraux. Cependant, des effets secondaires potentiellement sévères à long terme en limitent l'utilisation et doivent guider le choix thérapeutique. L'amélioration de la compréhension des mécanismes physiopathologiques impliqués dans la maladie conduit à l'identification de nouvelles molécules ciblant la composante inflammatoire, mais aussi la remyélinisation et la neuroprotection. <

La sclérose en plaques (SEP), affection démyélinisante inflammatoire du système nerveux central (SNC), fait intervenir des mécanismes auto-immuns complexes, associant lymphocytes T, lymphocytes B, cellules microgliales et cytokines, qui conduisent à une démyélinisation et une dégénérescence axonale (et neuronale), substrat de l'apparition et de l'aggravation d'un handicap irréversible. Actuellement, les traitements de fond disponibles (disease modifying therapies) ont pour action de limiter la réaction inflammatoire lymphocytaire. Ils permettent une réduction de la fréquence des poussées de la maladie, dans les formes rémittentes. En revanche, aucun traitement n'a, à ce jour, montré d'efficacité dans les phases progressives de la maladie. Leur indication est donc limitée aux formes évoluant par poussées. Les molécules thérapeutiques en développement ont pour objectif d'optimiser la stratégie anti-inflammatoire, mais aussi de favoriser la remyélinisation et la neuroprotection.

Depuis l'autorisation de mise sur le marché (AMM) du premier interféron $\beta$ lb il y a 20 ans, nous disposons de sept molécules approuvées en Europe (Tableau I) et huit aux États-Unis.

Vignette (Photo @ Inserm - Jean-Michel Peyrin).

\section{Nouveautés thérapeutiques et stratégies émergentes dans la sclérose en plaques}

Céline Louapre, Élisabeth Maillart, Caroline Papeix, Catherine Lubetzki

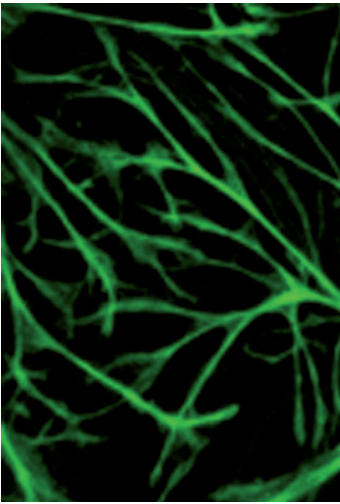

Département des maladies du système nerveux, Inserm 975 , hôpital Pitié-Salpêtrière, 47-83, boulevard de l'Hôpital, 75651 Paris Cedex 13, France. catherine.lubetzki@psl.aphp.fr

Les traitements de première ligne : traitement de référence pour les formes rémittentes de SEP

En première ligne, les immunomodulateurs (interféron $\beta$ : Betaferon ${ }^{\oplus}$, Avonex $^{\oplus}$, Rebif ${ }^{\oplus}$, Extavia $^{\oplus}$; et acétate de glatiramère : Copaxone ${ }^{\oplus}$ ) restent le traitement de référence pour les SEP rémittentes. Ils atténuent l'activation des lymphocytes $T$, inhibent la production de cytokines Thl pro-inflammatoires, activent la sécrétion de cytokines Th2 anti-inflammatoires et/ou inhibent le passage de lymphocytes autoréactifs du sang vers le système nerveux central en modulant la perméabilité de la barrière hémato-encéphalique (Figure 1, étapes 3 et 4). Cependant, ces deux traitements se distinguent par leur mode d'action. L'interféron $\beta$, comme la protéine naturelle sécrétée par l'organisme en réponse à la présence d'un agent étranger, se lie à des récepteurs présents à la surface des lymphocytes et des macrophages, alors que le copolymère (acétate de glatiramère) favoriserait une réponse Th2 anti-inflammatoire par un mécanisme qui reste imparfaitement connu. L'efficacité de ces deux traitements est identique : ils diminuent d'environ $30 \%$ la fréquence des poussées et réduisent d'environ $60 \%$ le nombre de nouvelles lésions en IRM (imagerie par résonance magnétique nucléaire) [1].

\section{Les traitements de deuxième ligne : pour les formes actives de SEP}

Plusieurs traitements ont démontré une efficacité supérieure aux traitements de première ligne dans la prévention des poussées. Cepen- 


\begin{tabular}{|c|c|c|c|}
\hline Traitement & $\begin{array}{l}\text { Doses/voie } \\
\text { d'administration }\end{array}$ & Surveillance & $\begin{array}{l}\text { Effets secondaires } \\
\text { principaux }\end{array}$ \\
\hline IFN- $\beta$ la $\left(\right.$ Avonex $\left.{ }^{\circledast}\right)$ & $\begin{array}{l}30 \mu \mathrm{g} / \mathrm{semaine} \\
\text { intramusculaire }\end{array}$ & & Syndrome pseudo-grippal, \\
\hline IFN- $\beta$ la $\left(\right.$ Rebif $\left.^{\boxplus}\right)$ & $\begin{array}{l}22 \text { ou } 44 \mu g \text { fois par } \\
\text { semaine, sous-cutanée }\end{array}$ & NFS, enzymes hépatiques & $\begin{array}{l}\text { réaction cutanée au site } \\
\text { d'injection, élévation des }\end{array}$ \\
\hline IFN- $\beta$ lb (Betaféron ${ }^{\circledast}$, Extavia $^{\circledast}$ ) & $\begin{array}{l}250 \mu \text { g tous les } 2 \text { jours, } \\
\text { sous-cutanée }\end{array}$ & $\int$ & enzymes hépatiques \\
\hline $\begin{array}{l}\text { Acétate de glatiramère } \\
\left(\text { Copaxone }{ }^{\circledast}\right)\end{array}$ & 20 mg/j, sous-cutanée & - & $\begin{array}{l}\text { Réaction cutanée au site } \\
\text { d'injection, lipo-atrophie, } \\
\text { oppression thoracique }\end{array}$ \\
\hline Mitoxantrone (Novantrone ${ }^{\circledast}$ ) & $\begin{array}{l}12 \mathrm{mg} / \mathrm{m}^{2} \text { tous les } \\
\text { mois pendant } 6 \text { mois, } \\
\text { intraveineux }\end{array}$ & $\begin{array}{l}\text { NFS, enzymes hépatiques, عCBU, } \\
\text { FEVG }\end{array}$ & $\begin{array}{l}\text { Cardiotoxicité, leucémie, } \\
\text { infertilité, leucopénie, } \\
\text { infections }\end{array}$ \\
\hline Natalizumab (Tysabri ${ }^{\circledR}$ ) & $\begin{array}{l}300 \mathrm{mg} \text { tous les } 28 \\
\text { jours, intraveineux }\end{array}$ & $\begin{array}{l}\text { NFS, enzymes hépatiques, } \\
\text { sérologie JC }\end{array}$ & LEMP, allergie (rare) \\
\hline Fingolimod (Gilenya ${ }^{\oplus}$ ) & $0,5 \mathrm{mg} / \mathrm{j}$, voie orale & $\begin{array}{l}\text { NFS, enzymes hépatiques, } \\
\text { surveillance ophtalmologique, } \\
\text { suivi cardiaque à l'introduction }\end{array}$ & $\begin{array}{l}\text { BAV et bradycardie à } \\
\text { l'introduction, œdème } \\
\text { maculaire, cytolyse hépatique }\end{array}$ \\
\hline
\end{tabular}

Tableau I. Traitements actuels pour la sclérose en plaques approuvés par l'agence européenne du médicament. NFS : numération formule sanguine ; $\varepsilon C B U$ : examen cytobactériologique urinaire; FEVG : fraction d'éjection du ventricule gauche ; LEMP : leuco-encéphalopathie multifocale progressive ; BAV : bloc auriculo-ventriculaire.

dant, leurs effets secondaires, parfois graves, limitent leur indication aux formes très actives de SEP et justifient une surveillance étroite. La mitoxantrone $\left(\varepsilon l s e p^{\circledR}\right)$ est le premier traitement à avoir été approuvé en deuxième intention. II s'agit d'une molécule antinéoplasique de la famille des anthracyclines qui inhibe la topo-isomérase de type II. La mitoxantrone est utilisée dans la SEP comme agent immunosuppresseur. Plusieurs études ont montré qu'elle réduisait le nombre de poussées, l'apparition de nouvelles lésions rehaussées par le gadolinium (ce qui traduit une rupture de la barrière hémato-encéphalique) ${ }^{1}$ et la progression du handicap [2-4]. L'efficacité de la mitoxantrone se maintient plusieurs mois après l'arrêt du traitement. II s'agit du seul traitement d'induction dans la SEP disponible aujourd'hui dans les formes très actives de la maladie. Cependant, les effets secondaires (leucémie et insuffisance cardiaque), bien que rares, peuvent survenir également après l'arrêt du traitement et doivent être dépistés de façon systématique pendant cinq ans.

Le natalizumab (Tysabri ${ }^{\oplus}$, 2007) est un anticorps monoclonal humanisé dirigé contre l'intégrine $\alpha 4$ (VLA4), exprimée à la surface des lymphocytes activés et des monocytes [25]. Cette molécule est un ligand des molécules d'adhésion (VCAM, vascular cell adhesion molecule) exprimées sur l'endothélium. En bloquant l'interaction

\footnotetext{
${ }^{1}$ Le gadolinium est le produit de contraste utilisé en IRM, qui, en cas de rupture de la barrière hématoencéphalique au niveau des lésions récentes (qu'on appelle actives), fait apparaître un «hypersignal » sur l'IRM, d'où le terme de « rehaussées ».
}

VLA4/VCAM, le natalizumab inhibe la fixation des lymphocytes activés sur l'endothélium, et empêche ainsi le passage de ces cellules immuno-compétentes à travers la barrière hémato-encéphalique, et donc leur entrée dans le système nerveux central (Figure l, étape 3). L'administration intraveineuse de natalizumab diminue la fréquence annualisée des poussées d'environ $60 \%$ et réduit d'environ $90 \%$ le nombre de nouvelles lésions actives à I'IRM [5]. Les résultats des essais thérapeutiques ont objectivé par rapport au placebo une diminution d'environ $40 \%$ du risque d'aggravation du handicap neurologique à deux ans. Cependant des cas de leuco-encéphalopathie multifocale progressive (LEMP, réactivation d'une infection à polyomavirus JC) ont été rapportés chez des patients sous natalizumab, avec plusieurs centaines de cas dans le monde depuis l'initiation du traitement. Ceci pose le problème du rapport bénéfice/risque du natalizumab. II s'agit en effet d'une complication extrêmement sévère, vraisemblablement secondaire à l'immunosuppression profonde et prolongée du système nerveux central, et qui peut être mortelle ou entraîner des séquelles neurologiques majeures. Nos connaissances sur l'incidence de la LEMP sous natalizumab se sont affinées depuis peu, permettant 


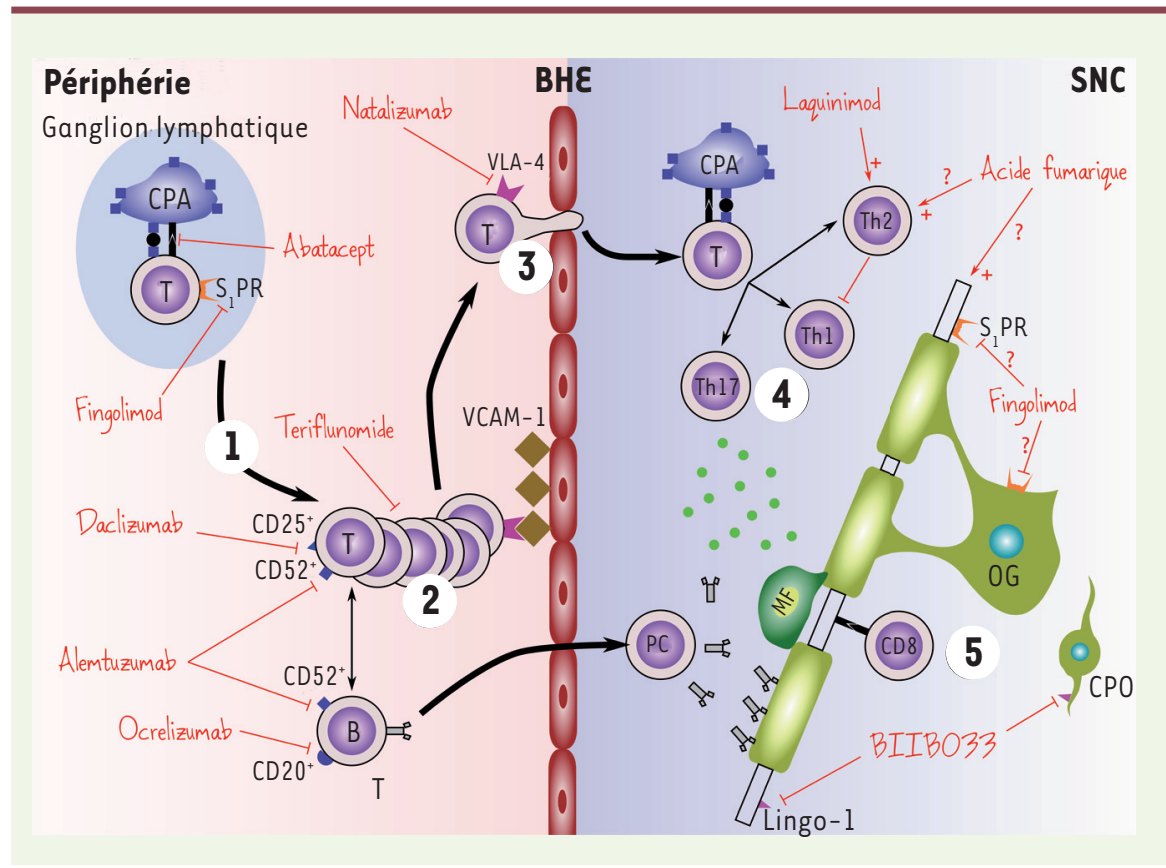

Figure 1. Physiopathologie de la SEP et cibles des nouveaux traitements. Principales étapes de la physiopathologie de la sclérose en plaques et cibles thérapeutiques : (1) présentation de l'antigène au lymphocyte T, sortie du ganglion lymphatique ; (2) prolifération lymphocytaire ; (3) passage de la barrière hémato-encéphalique (BHE); (4) réaction immunitaire spécifique avec activation des Thl et Th17 et sécrétion de cytokines pro-inflammatoires, activation des lymphocytes CD8 cytotoxiques aboutissant à une destruction de la myéline et, éventuellement, de l'axone; et (5) remyélinisation et protection axonale. CPA : cellule présentatrice d'antigènes ; $O G$ : oligodendrocyte ; $C P O$ : cellule précurseur d'oligodendrocyte; BHE : barrière hémato-encéphalique; SIPR : récepteur de la sphingosine 1-phosphate ; SNC : système nerveux central ; Mab : monoclonal antibody. Les données ont été extraites de [24]. récepteurs sphingosine 1 -phosphate (SIPl à S1P5) [26]. Cette famille de récepteurs a une expression ubiquitaire dans l'organisme, notamment dans le système immunitaire et le cerveau. La liaison aux récepteurs exprimés par les lymphocytes conduit ces derniers à être «séquestrés» dans les organes lymphoïdes, ce qui est à l'origine de la lymphopénie périphérique et d'une diminution du nombre de lymphocytes pénétrant dans le système nerveux central (Figure 1, étape 1). Ce traitement oral (un comprimé de 0,5 mg par jour) diminue la fréquence annualisée des poussées d'environ $55 \%$ [6]. Le profil de tolérance n'est pas le même: le risque de survenue d'une bradycardie (chute de 8 battements par minute en moyenne), voire d'un bloc auriculo-ventriculaire lors de l'instauration du fingolimod, nécessite un suivi cardiaque en milieu hospitalier lors de la première prise du médicament. La survenue potentielle de complications infectieuses, notamment la réactivation de virus du groupe herpès, justifie un suivi régulier. une relative «stratification du risque ». Si l'incidence globale de la LEMP chez les patients traités par natalizumab est estimée à deux cas pour 1000 patients, l'analyse rétrospective des cas de LEMP a montré que ce risque varie en fonction de plusieurs facteurs: (1) le statut sérologique vis-à-vis du virus JC; (2) une durée de traitement supérieure à 24 mois; et (3) l'exposition antérieure à un traitement immuno-suppresseur. Chez les patients ne présentant aucun de ces facteurs de risque, le risque de survenue d'une LEMP est de 0,1/1000. $\varepsilon n$ revanche, lorsque les trois facteurs sont réunis, il est de $1 / 100$ [6]. Ces chiffres sont néanmoins susceptibles de se modifier avec l'augmentation du nombre de patients traités.

Les indications du natalizumab retenues en France pour l'AMM sont:

- en première ligne, les formes sévères et d'évolution rapide, définies par deux poussées invalidantes ou plus au cours d'une année, associées à une ou plusieurs lésion(s) rehaussée(s) après injection de gadolinium sur l'IRM cérébrale ou à une augmentation significative de la charge lésionnelle en T2 par rapport à une IRM antérieure récente ;

- en deuxième ligne, les formes très actives de la maladie, malgré un traitement par l'interféron $\beta$.

Avec une AMM identique à celle du natalizumab, le fingolimod (Gilenya ${ }^{\circledR}, 2011$ ) constitue actuellement une alternative thérapeutique en deuxième intention. Cet immunosuppresseur sélectif est un ligand des

\section{Les perspectives thérapeutiques à court terme}

Les traitements actifs sur la composante inflammatoire actuellement à l'étude agissent sur différentes étapes de la cascade immunologique de la maladie (Tableau II).

Les nouveaux immunomodulateurs par voie orale Le tériflunomide (Aubagio ${ }^{\oplus}$ ) est un traitement approuvé en septembre 2012 par la Food and drug administration (FDA) aux États-Unis. II inhibe de façon réversible une enzyme mitochondriale clé impliquée dans la synthèse des bases pyrimidiques pour la réplication de I'ADN, et réduit ainsi l'activation et la prolifération des lymphocytes T et B (Figure 1, étape 2). À la dose de $14 \mathrm{mg}$ par jour, il diminue le risque annualisé de poussées de $31 \%$ par rapport au placebo [7].

L'acide diméthylfumarique active la voie Nrf 2 (nuclear factor [erythroid-derived 2]-like 2), favorisant une réduction de la production des radicaux libres. II entraînerait aussi une diminution des cytokines proinflammatoires (TNF $\alpha$ [tumor necrosis factor $\alpha$ ], 


\begin{tabular}{|c|c|c|c|c|c|}
\hline Molécules & $\begin{array}{l}\text { Mécanismes } \\
\text { d'actions }\end{array}$ & Phase & Administration & $\begin{array}{c}\text { Résultats } \\
\text { (versus } \\
\text { placebo ou } \\
\text { comparateur) }\end{array}$ & $\begin{array}{c}\text { Effets } \\
\text { secondaires principaux }\end{array}$ \\
\hline Laquinimod & $\begin{array}{l}\text { Favorise le passage } \\
\text { des lymphocytes } \\
\text { pro-inflammatoires } \\
\text { Th1 vers un profil } \\
\text { Th2 ; pourrait } \\
\text { favoriser la } \\
\text { sécrétion d'agents } \\
\text { neuroprotecteurs }\end{array}$ & III & $\begin{array}{l}0,6 \mathrm{mg} / \mathrm{j}, \\
\text { voie orale }\end{array}$ & $\begin{array}{l}\text { Réduction de } 23 \% \\
\text { de la fréquence des } \\
\text { poussées, réduction } \\
\text { de } 37 \% \text { des lésions } \\
\text { IRM actives }\end{array}$ & $\begin{array}{l}\text { Élévation des enzymes } \\
\text { hépatiques, réactivations } \\
\text { herpétiques }\end{array}$ \\
\hline Teriflunomide & $\begin{array}{l}\text { Inhibe la synthèse } \\
\text { des pyrimidines en } \\
\text { particulier dans les } \\
\text { cellules B et T en } \\
\text { mitose }\end{array}$ & III & $\begin{array}{l}7 \text { et } 14 \mathrm{mg} / \mathrm{j} \text {, } \\
\text { voie orale }\end{array}$ & $\begin{array}{l}\text { Réduction de } 31 \% \\
\text { du risque annualisé } \\
\text { de poussée, } \\
\text { diminution de } 61 \% \\
\text { des lésions IRM } \\
\text { actives, réduction } \\
\text { de la progression du } \\
\text { handicap? }\end{array}$ & $\begin{array}{l}\text { Troubles gastro-intestinaux, } \\
\text { alopécie, rash cutané, } \\
\text { infections, tératogénicité }\end{array}$ \\
\hline $\begin{array}{l}\text { Acide } \\
\text { diméthylfumarique } \\
\text { (BGl2) }\end{array}$ & $\begin{array}{l}\text { Active la voie du } \\
\text { Nrf2, anti-oxydant } \\
\text { cellulaire }\end{array}$ & III & $\begin{array}{l}120 \text { à } 240 \mathrm{mg} \\
3 \text { fois } / \mathrm{j}, \\
\text { voie orale }\end{array}$ & $\begin{array}{l}\text { Réduction de } \\
53 \% \text { du risque } \\
\text { annualisé de } \\
\text { poussée, réduction } \\
\text { de la progression du } \\
\text { handicap à } 2 \text { ans de } \\
49 \%\end{array}$ & $\begin{array}{l}\text { Céphalées, flush, troubles } \\
\text { gastro-intestinaux, élévation } \\
\text { des enzymes hépatiques }\end{array}$ \\
\hline Alemtuzumab & Anticorps anti-CD52 & III & $\begin{array}{l}12 \mathrm{mg} / \mathrm{j} \text { pendant } \\
5 \text { jours, } 12 \mathrm{mg} / \mathrm{j} \\
\text { pendant } 3 \text { jours } \\
\text { un an après, voie } \\
\text { intraveineuse }\end{array}$ & $\begin{array}{l}\text { Réduction de } 55 \% \\
\text { du taux annualisé } \\
\text { de poussée par } \\
\text { rapport à l'IFN- } \\
\text { Bla, réduction de } \\
\text { la progression du } \\
\text { handicap? }\end{array}$ & $\begin{array}{l}\text { Purpura thrombopénique } \\
\text { auto-immun, thyroïdite auto- } \\
\text { immune }\end{array}$ \\
\hline Daclizumab & $\begin{array}{l}\text { Anticorps anti-CD25 } \\
\text { /récepteur de l'IL2 }\end{array}$ & II & $\begin{array}{l}2 \mathrm{mg} / \mathrm{kg} / 2 \text { semaines } \\
\text { en sous-cutané, ou } \\
150 \text { à } 300 \mathrm{mg} \text { en } \\
\text { intraveineux toutes } \\
\text { les } 4 \text { semaines }\end{array}$ & $\begin{array}{l}\text { Réduction de } 72 \% \\
\text { des lésions IRM } \\
\text { actives, réduction } \\
\text { de } 50 \% \text { du taux } \\
\text { annualisé de } \\
\text { poussée }\end{array}$ & $\begin{array}{l}\text { Rash cutanés, } \\
\text { lymphadénopathies, élévation } \\
\text { des enzymes hépatiques }\end{array}$ \\
\hline Ocrélizumab & Anticorps anti-CD20 & II & $\begin{array}{l}600 \text { à } 2000 \mathrm{mg} \\
\text { initialement puis } \\
600 \text { à } 1000 \mathrm{mg} \text { à } \\
24 \text { semaines, voie } \\
\text { intraveineuse }\end{array}$ & $\begin{array}{l}\text { Réduction de } 89 \text { à } \\
96 \% \text { des lésions IRM } \\
\text { actives }\end{array}$ & $\begin{array}{l}\text { Infections opportunistes, } \\
\text { réaction inflammatoire } \\
\text { systémique }\end{array}$ \\
\hline
\end{tabular}

Tableau II. Principaux traitements en développement dans la sclérose en plaques et résultats issus de différents essais cliniques. Les résultats obtenus pour chacune des molécules (réduction de la fréquence des poussées et de l'apparition de nouvelles lésions IRM) ne peuvent pas être comparés entre eux, car issus d'essais cliniques différents. 
IL [interleukine] $1 \beta$ et IL6) avec une diminution de l'activation de la microglie. Il diminue le risque de poussées de $53 \%$ et le nombre de nouvelles lésions de $80 \%$ [8].

Le laquinimod agirait en diminuant l'expression des facteurs de transcription impliqués dans la régulation du gène de CMH (complexe majeur d'histocompatibilité) de classe II et en activant, par la voie de I'IL4, les propriétés anti-inflammatoires des lymphocytes CD4, ainsi que l'apoptose des lymphocytes T CD8 et B. C'est un traitement oral bien toléré, mais l'efficacité clinique est modeste [9].

\section{Les anticorps monoclonaux}

L'alemtuzumab (MabCampath $^{\circledR}$ ) est un anticorps monoclonal humanisé anti-CD52, induisant une déplétion très profonde et prolongée des lymphocytes B et T. Des effets secondaires (purpura thrombopénique, thyroïdites, syndrome de Goodpasture) ont été signalés. En première ligne de traitement, il diminue le risque de poussées de $55 \%$ par rapport à l'interféron $\beta[10,11]$.

Le daclizumab est un anticorps monoclonal neutralisant dirigé contre le récepteur de l'IL2 (chaîne $\alpha$ CD25). Il inhibe la costimulation nécessaire à la prolifération des lymphocytes T et augmente l'activité d'une population de cellules régulatrices (cellules natural killer NK). Des études de phase III sont en cours.

L'ocrelizumab est un anticorps monoclonal humanisé anti-CD20 qui induit une déplétion lymphocytaire $B$. Les résultats de l'étude de phase II, en montrant une réduction majeure de l'activité IRM et une réduction de la fréquence des poussées, ont démontré l'importance de la réaction lymphocytaire B dans la SEP [12]. Deux essais de phase III sont actuellement en cours dans les formes rémittente et progressive de la maladie.

\section{Les molécules candidates et les stratégies thérapeutiques de demain}

Parmi les molécules candidates pour des essais cliniques futurs, certaines ciblent les mécanismes physiopathologiques déjà connus, en visant une meilleure sécurité d'emploi; d'autres ciblent des mécanismes qui permettraient une meilleure efficacité dans les formes progressives.

Deux molécules ont pour cible des interactions déjà utilisées dans les traitements disponibles: il s'agit de l'ELND002, qui agirait au même niveau que le natalizumab, en bloquant l'intégrine $\alpha 4$ et qui s'administre en injection sous-cutanée (phase I en cours); et du BAF312 qui est un ligand des récepteurs du SIP, mais qui ne cible qu'un nombre restreint de ces récepteurs (SIPI-SIP5), dans l'objectif de limiter certains effets secondaires. Néanmoins, l'absence d'effets cardiovasculaires rapportée chez l'animal n'a pas été confirmée chez l'homme, puisqu'une bradycardie transitoire a été observée lors de la phase I [13].

Le masitinib est un inhibiteur de tyrosine kinase qui a pour cible les mastocytes, cellules impliquées dans les réactions allergiques et inflammatoires. Une étude de phase $\mathrm{Ilb} / \mathrm{III}$ est en cours dans les formes progressives.
L'abatacept est une molécule utilisée dans la polyarthrite rhumatoïde qui mime l'activité du CTLA-4 (cytotoxic T-lymphocyte antigen 4) et empêche l'interaction entre les cellules présentatrices d'antigènes et les lymphocytes T [27]. Une étude de phase II est en cours dans les SEP rémittentes.

D'autres stratégies immunomodulatrices sont envisagées: 1. Les approches de tolérisation sont basées sur le principe de l'administration d'antigènes myéliniques afin d'«éduquer » le système immunitaire et d'induire un état de non-réponse spécifique à l'antigène. Bien que les essais chez l'homme aient été décevants dans le passé $[14,15]$, des travaux récents sur des modèles animaux de SEP ont permis d'améliorer la tolérance immunitaire grâce à l'administration d'épitopes plus ciblés [16-18].

2. Les approches centrées sur l'hypothèse rétrovirale: l'implication d'un rétrovirus endogène (multiple sclerosis-associated retrovirus, MSRV) dans la SEP est suggérée depuis de nombreuses années [19]. La protéine d'enveloppe du virus pourrait activer une cascade proinflammatoire par le biais de son interaction avec les récepteurs toll-like. Ces travaux ont conduit à l'initiation récente d'un essai clinique (phase I et Ila) chez les patients atteints de SEP, avec un anticorps monoclonal dirigé contre cette protéine d'enveloppe [20].

Les stratégies de remyélinisation et de neuroprotection ont pour objectif de protéger l'axone, dont l'atteinte, en grande partie secondaire à une démyélinisation prolongée, est précoce dans la maladie [21]. Elles comportent deux aspects :

- la remyélinisation par voie endogène : il s'agit d'amplifier les capacités de réparation spontanée de la myéline ; - la remyélinisation par voie exogène [22], c'est-à-dire la greffe de cellules potentiellement myélinisantes. Différents types cellulaires sont évalués. Les cellules souches mésenchymateuses semblent avoir essentiellement un effet immuno-modulateur (étude européenne en cours). Les stratégies utilisant des cellules « réparatrices » restent du domaine de la recherche expérimentale: cellules olfactives engainantes, cellules souches neurales ou progéniteurs oligodendrogliaux, et cellules souches pluripotentes induites (iPS cells).

Les mécanismes cellulaires et moléculaires qui soustendent le processus de remyélinisation endogène sont mieux connus, ce qui a conduit à l'identification de facteurs activateurs et inhibiteurs. Lingo-l est l'un de ces facteurs inhibiteurs; son expression par les cellules oligodendrogliales immatures empêche leur différenciation. Dans des modèles expérimentaux, le blocage de Lingo-1 favorise la myélinisation et la remyélinisation. Ces résultats précliniques ont conduit à un développement translationnel avec un anticorps monoclonal 
anti-Lingo-1 humanisé. L'étude de phase I est terminée et deux études de phase II viennent de débuter. II s'agit de la première étude ciblant la remyélinisation dans la SEP [23].

En dehors de la remyélinisation qui permet de prévenir la neurodégénérescence, aucune molécule thérapeutique n'a encore convaincu d'une efficacité neuroprotectrice.

\section{Conclusion}

L'apparition de nouveaux traitements plus efficaces représente un progrès considérable dans le traitement de la sclérose en plaques évoluant par poussées. Néanmoins, ces nouvelles thérapeutiques ont accru la complexité de la prise en charge individuelle des patients. Ces nouveaux traitements diffèrent par leur voie d'administration, leur mécanisme d'action, leur efficacité, mais aussi leur sécurité d'emploi. L'algorithme qui permettrait, en tenant compte de différents paramètres, de sélectionner la stratégie thérapeutique optimale parmi ces différents traitements reste encore à définir. Les résultats des études portant sur l'effet des immunothérapies sur la phase progressive de la maladie sont attendus dans les prochaines années. Les stratégies de remyélinisation et de neuroprotection sont également un défi majeur dans les années à venir pour éviter ou réparer les lésions tissulaires. $\diamond$

\section{SUMMARY}

\section{New and emerging treatments for multiple sclerosis}

A number of disease-modifying therapies have been recently approved for the treatment of multiple sclerosis (MS). These molecules which prevent relapses and new central nervous system lesions are more efficient than the "old" first line therapies and/or more convenient, especially with oral agents. Their usefulness in treating active MS is undeniable, but we have to be aware of their potentially extremely severe side effects. The treatment algorithm in MS is constantly evolving according to the long term safety profile of these new treatments. A better knowledge of fundamental pathophysiology is associated with the development of new molecules targeting the immunological cascade of the disease as well as the mechanisms promoting remyelination and repair. $\diamond$

\section{LIENS D'INTÉRÊT}

Catherine Lubetzki déclare une participation ponctuelle à des conseils consultatifs pour Roche, Biogen, Novartis. Céline Louapre déclare avoir reçu ponctuellement des honoraires de Novartis pour une présentation. Caroline Papeix déclare avoir reçu ponctuellement des honoraires comme consultante ou pour des présentations de Bayer-Schering, Biogen Idec, Novartis, Merck Serono, Sanofi Aventis, Teva Pharma, Roche, Genzyme.

Tous les auteurs participent actuellement à des essais cliniques dans la SEP, concernant les laboratoires Roche, Genzyme, Biogen, Novartis.

\section{RÉFÉRENCES}

1. Cadavid D, Wolansky LJ, Skurnick J, et al. Efficacy of treatment of MS with IFNbeta-1b or glatiramer acetate by monthly brain MRI in the BECOME study. Neurology 2009 ; 72 : 1976-83.

2. Hartung HP, Gonsette R, Konig N, et al. Mitoxantrone in progressive multiple sclerosis: a placebocontrolled, double-blind, randomised, multicentre trial. Lancet $2002 ; 360: 2018-25$.

3. Edan G, Miller D, Clanet M, et al. Therapeutic effect of mitoxantrone combined with methylprednisolone in multiple sclerosis: a randomised multicentre study of active disease using MRI and clinical criteria. J Neurol Neurosurg Psychiatry 1997 ; 62 : 112-8.
4. Millefiorini $\varepsilon$, Gasperini C, Pozzilli C, et al. Randomized placebo-controlled trial of mitoxantrone in relapsing-remitting multiple sclerosis: 24 -month clinical and MRI outcome. J Neurol 1997 ; 244 : 153-9.

5. Polman $\mathrm{CH}, \mathrm{O}$ 'Connor PW, Havrdova $\varepsilon$, et al. A randomized, placebocontrolled trial of natalizumab for relapsing multiple sclerosis. $N$ Engl J Med $2006 ; 354: 899-910$.

6. Kappos L, Radue $\varepsilon W, O$ 'Connor P, et al. A placebo-controlled trial of oral fingolimod in relapsing multiple sclerosis. N Engl J Med 2010 ; 362 : 387-401.

7. O'Connor P, Wolinsky JS, Confavreux C, et al. Randomized trial of oral teriflunomide for relapsing multiple sclerosis. N Engl J Med 2011 ; 365 : 1293-303.

8. Gold R, Kappos L, Arnold DL, et al. Placebo-controlled phase 3 study of oral BG-12 for relapsing multiple sclerosis. N Engl J Med 2012 ; 367 : 1098-107.

9. Comi G, Jeffery D, Kappos L, et al. Placebo-controlled trial of oral laquinimod for multiple sclerosis. N Engl J Med 2012 ; 366 : 1000-9.

10. Papeix C, Lubetzki C. Anticorps monoclonaux dans la sclérose en plaques. Med Sci (Paris) $2009 ; 25: 1113-5$.

11. Cohen JA, Coles AJ, Arnold DL, et al. Alemtuzumab versus interferon beta la as first-line treatment for patients with relapsing-remitting multiple sclerosis: a randomised controlled phase 3 trial. Lancet $2012 ; 380$ : 1819-28.

12. Kappos L, Li D, Calabresi PA, et al. Ocrelizumab in relapsing-remitting multiple sclerosis: a phase 2, randomised, placebo-controlled, multicentre trial. Lancet $2011 ; 378: 1779-87$.

13. Gergely P, Nuesslein-Hildesheim B, Guerini D, et al. The selective sphingosine 1-phosphate receptor modulator BAF312 redirects lymphocyte distribution and has species-specific effects on heart rate. Br J Pharmacol 2012 ; 167 : 1035-47.

14. Weiner HL, Mackin GA, Matsui M, et al. Double-blind pilot trial of oral tolerization with myelin antigens in multiple sclerosis. Science $1993 ; 259$ : 1321-4.

15. Van $\operatorname{der}$ Aa A, Hellings N, Medaer R, et al. T cell vaccination in multiple sclerosis patients with autologous CSF-derived activated T cells: results from a pilot study. Clin Exp Immunol 2003 ; 131 : 155-68.

16. Mannie MD, Blanchfield JL, Islam SM, Abbott DJ. Cytokine-neuroantigen fusion proteins as a new class of tolerogenic, therapeutic vaccines for treatment of inflammatory demyelinating disease in rodent models of multiple sclerosis. Front Immunol $2012 ; 3: 255$.

17. Kaushansky N, Kerlero de Rosbo N, Zilkha-Falb R, et al. Multi-epitopetargeted immune-specific therapy for a multiple sclerosis-like disease via engineered multi-epitope protein is superior to peptides. PLoS One $2011 ; 6$ : e27860.

18. Billetta R, Ghahramani N, Morrow 0, et al. Epitope-specific immune tolerization ameliorates experimental autoimmune encephalomyelitis. Clin Immunol $2012 ; 145$ : 94-101.

19. Perron H, Garson JA, Bedin F, et al. Molecular identification of a novel retrovirus repeatedly isolated from patients with multiple sclerosis. The collaborative research group on multiple sclerosis. Proc Natl Acad Sci USA $1997 ; 94: 7583-8$.

20. Curtin F, Lang AB, Perron $\mathrm{H}$, et al. GNbACl, a humanized monoclonal antibody against the envelope protein of multiple sclerosis-associated endogenous retrovirus: a first-in-humans randomized clinical study. Clin Ther $2012 ; 34: 2268-78$.

21. Lubetzki C. Sclérose en plaques : quelles possibilités de régénération ? Bull Acad Natl Med 2008 ; 192 : 495-506.

22. Lubetzki C, Williams A, Stankoff B. Promoting repair in multiple sclerosis: problems and prospects. Curr Opin Neurol 2005 ; 18 : 237-44.

23. Mi S, Miller RH, Tang W, et al. Promotion of central nervous system remyelination by induced differentiation of oligodendrocyte precursor cells. Ann Neurol 2009 ; 65 : 304-15.

24. Linker RA, Kieseier BC, Gold R. Identification and development of new therapeutics for multiple sclerosis. Trends Pharmacol Sci 2008 ; 29 : 558-65.

25. Papeix C, Lubetzki C. Anticorps monoclonaux dans la sclérose en plaques. Med Sci (Paris) $2009 ; 25: 1113-5$.

26. Cuvillier 0 . Les récepteurs de la sphingosine 1-phosphate. Med Sci (Paris) $2012 ; 28: 951-7$.

27. Sibilia J. Protéine de fusion ou anticorps monoclonal. Med Sci (Paris) 2009 ; $25: 1033-8$.
TIRÉS À PART

C. Louapre 